\title{
IMPROVED BEREZIN-LI-YAU INEQUALITIES WITH MAGNETIC FIELD
}

\author{
HYNEK KOVAŘÍK AND TIMO WEIDL
}

\begin{abstract}
In this paper we study the eigenvalue sums of Dirichlet Laplacians on bounded domains. Among our results we establish an improvement of the Li-Yau bound in the presence of a constant magnetic field previously obtained in [5].
\end{abstract}

\section{Introduction}

Let $\Omega \subset \mathbb{R}^{\mathrm{d}}$ be an open bounded domain. We consider the Dirichlet Laplacian $-\Delta_{\Omega}$ on $\mathrm{L}^{2}(\Omega)$ defined in the quadratic form sense. Since the embedding $\mathrm{H}_{0}^{1} \hookrightarrow \mathrm{L}^{2}(\Omega)$ is compact, the spectrum of the non-negative operator $-\Delta_{\Omega}$ is discrete and accumulates to infinity only. Denote by $\left\{\lambda_{j}\right\}_{j \in \mathbb{N}}=$ $\left\{\lambda_{j}(\Omega)\right\}_{j \in \mathbb{N}}$ the inreasing sequence of the eigenvalues of $-\Delta_{\Omega}$, where we repeat entrees according to their multiplicity.

In particular, we shall study the so-called Riesz means of these eigenvalues, given by 1

$$
\operatorname{tr}\left(-\Delta_{\Omega}-\Lambda\right)_{-}^{\gamma}=\sum_{k}\left(\Lambda-\lambda_{k}\right)_{+}^{\gamma}, \quad \gamma \geq 0
$$

Here and below we use the notation $x_{ \pm}=(|x| \pm x) / 2$. It is well-known that these Riesz means satisfy the Weyl asymptotics [24]

$$
\sum_{k}\left(\Lambda-\lambda_{k}\right)_{+}^{\gamma}=L_{\gamma, d}^{c l}|\Omega| \Lambda^{\gamma+\frac{d}{2}}+o\left(\Lambda^{\gamma+\frac{d}{2}}\right), \quad \Lambda \rightarrow \infty
$$

where

$$
\mathrm{L}_{\gamma, \mathrm{d}}^{\mathrm{cl}}=\frac{\Gamma(\gamma+1)}{(4 \pi)^{\frac{d}{2}} \Gamma(\gamma+1+\mathrm{d} / 2)} .
$$

In 1972 Berezin [2] showed that for $\gamma \geq 1$ the leading term in (1.1) gives actually a uniform upper bound on the Riesz means, namely for any $\gamma \geq 1$ it holds

$$
\sum_{k}\left(\Lambda-\lambda_{k}\right)_{+}^{\gamma} \leq \mathrm{L}_{\gamma, \mathrm{d}}^{\mathrm{cl}}|\Omega| \Lambda^{\gamma+\frac{\mathrm{d}}{2}}
$$

In view of the asymptotics $(1.1)$ the constant on the r.h.s. of (1.2) is optimal. The bound (1.2) is assumed to hold for all $0 \leq \gamma<1$ as well. However, so far this has been shown for tiling domains [21] and cartesian products with tiling domains [14] only. On the other hand, it follows from (1.2) that a similar inequality holds for arbitrary domains and for all $0 \leq \gamma<1$ with some probably non-sharp excess factor on the r.h.s. [14]

$$
\sum_{k}\left(\Lambda-\lambda_{k}\right)_{+}^{\gamma} \leq 2\left(\frac{\gamma}{\gamma+1}\right)^{\gamma} \mathrm{L}_{\gamma, d}^{c l}|\Omega| \Lambda^{\gamma+\frac{d}{2}}, \quad 0 \leq \gamma<1 .
$$

\footnotetext{
${ }^{1}$ For $\gamma=0$ this is simply the counting function of all eigenvalues $\lambda_{j}(\Omega)<\Lambda$.
} 
Here we are going to focus on the border-line case $\gamma=1$, in which the inequality (1.2) is equivalent, via Legendre transformation, to the lower bound

$$
\sum_{j=1}^{N} \lambda_{j}(\Omega) \geq C_{d}|\Omega|^{-\frac{2}{d}} N^{1+\frac{2}{d}}, \quad C_{d}=\frac{4 \pi d}{d+2} \Gamma(d / 2+1)^{\frac{2}{d}} .
$$

The above estimate was proved in [17] independently on [2] and it is known as the Li-Yau inequality. Similarly as in the case of Berezin inequality, the constant $C_{d}$ cannot be improved, since the right hand side of (1.4) gives the leading term of the Weyl asymtotic formula, see (2.8) below.

However, the bounds (1.2) and (1.4) can be improved by adding to its right hand side reminder term of a lower order in $\Lambda$ or in $N$, respectively. Several results in this direction were obtained recently both for the Berezin inequality [9, 23] (for $\gamma \geq \frac{3}{2}$ ) and for the Li-Yau estimate [8, 20, 13, 25. 26]. In particular, Melas proved in [20] that there exists a positive constant $M_{d}$ such that

$$
\sum_{j=1}^{N} \lambda_{j}(\Omega) \geq C_{d}|\Omega|^{-\frac{2}{d}} N^{1+\frac{2}{d}}+M_{d} \frac{|\Omega|}{I(\Omega)} N, \quad I(\Omega)=\min _{a \in \mathbb{R}^{d}} \int_{\Omega}|x-a|^{2} d x,
$$

where $M_{d} \geq \frac{1}{24(d+2)}$.

Alongside with the ordinary Dirichlet Laplacian we shall also consider its magnetic version $H(A)=(i \nabla+A(x))^{2}$ on $L^{2}(\Omega)$ generated by the closed quadratic form

$$
\|(i \nabla+A) u\|_{L^{2}(\Omega)}^{2}, \quad u \in H_{0}^{1}(\Omega),
$$

where $A$ is a real-valued vector potential satisfying mild regularity conditions. Moreover, the magnetic Sobolev norm on the bounded domain $\Omega$ is equivalent to the non-magnetic one and the operator $H(A)$ has discrete spectrum as well. We notate its eigenvalues by $\lambda_{k}=\lambda_{k}(\Omega ; A)$, repeating eigenvalues according to their multiplicities. Note that the magnetic Riesz means satisfy the very same Weyl asymptotics (1.1).

From the pointwise diamagnetic inequality (see e.g. [18, Thm.7.21])

$$
|\nabla| u(x)|| \leq|(i \nabla+A) u(x)| \quad \text { a. e. } \quad x \in \Omega,
$$

it follows that $\lambda_{1}(\Omega ; A) \geq \lambda_{1}(\Omega ; 0)=\lambda_{1}(\Omega)$. However, the estimate $\lambda_{j}(\Omega ; A) \geq \lambda_{j}(\Omega ; 0)=\lambda_{j}(\Omega)$ fails in general if $j \geq 2$. Therefore, it is a priori not clear whether bounds similar to (1.2)-(1.5) remain true when the eigenvalues $\lambda_{j}(\Omega)$ are replaced by their magnetic counterparts $\lambda_{j}(\Omega ; A)$.

By now it has been shown that

- the sharp bound (1.2) holds true for arbitrary magnetic fields if $\gamma \geq \frac{3}{2}([15])$,

- the sharp bound (1.2) holds true for constant magnetic fields if $\gamma \geq 1$ ([5]),

- in the dimension $d=2$ the bound (1.3) holds true for constant magnetic fields if $0 \leq \gamma<1$ and the constant on the r.h.s. of (1.3) cannot be improved ([7]) even in the class of constant magnetic fields and tiling domains $\Omega$.

So far it is not known, whether the bound (1.2) holds true for arbitrary magnetic fields if $1 \leq \gamma \leq \frac{3}{2}$.

For $\gamma=1$ and constant magnetic field the magnetic version of (1.2) is again dual to the magnetic version of the Li-Yau bound (1.4). Since (1.2) fails without excess factor for all $\gamma<1$, the case $\gamma=1$ is the threshold case, in which the Berezin bound with the classical constant remains true. Therefore it is of a particular interest to study, whether either the magnetic Berezin bound for $\gamma=1$ or equivalently the magnetic Li-Yau bound admits any further improvement by lower order remainder terms. 
The main purpose of this paper is to establish an improved Li-Yau bound with an additional term of the Melas order for magnetic Dirichlet Laplacians on planar domains $\Omega \subset \mathbb{R}^{2}$ with constant magnetic field. For this end we first prove a different version of the Melas result in the non-magnetic case. Our proof is based on a new approach and yields the reminder term of the same order in $\mathrm{N}$, i.e. linear, but with a different geometrical factor, see Theorem 2.3 and Corollary 2.4 More importantly, in contrast to the classical Melas proof our method extends to a lower bound for the magnetic eigenvalues $\lambda_{k}(\Omega ; A)$ as well, see Theorem 2.7 and Corollary 2.8

\section{Main results}

2.1. Preliminaries. Given a set $\Omega \subset \mathbb{R}^{\mathrm{d}}$ we denote its volume by $|\Omega|$. Moreover, we denote by

$$
\delta(x)=\operatorname{dist}(x, \partial \Omega)=\min _{y \in \partial \Omega}|x-y|
$$

the distance between a given $x \in \Omega$ and the boundary of $\Omega$, and by

$$
R_{i}(\Omega)=\sup _{x \in \Omega} \delta(x)
$$

the in-radius of $\Omega$. Given $\beta>0$ we introduce

$$
\Omega_{\beta}=\{x \in \Omega: \delta(x)<\beta\}, \quad \beta>0,
$$

and define the quantity

$$
\sigma(\Omega):=\inf _{0<\beta<R_{i}(\Omega)} \frac{\left|\Omega_{\beta}\right|}{\beta} .
$$

Note that $\sigma(\Omega)>0$ since the right hand side of (2.2) is a positive continuous function of $\beta$ and

$$
\liminf _{\beta \rightarrow 0} \frac{\left|\Omega_{\beta}\right|}{\beta}>0 \text {. }
$$

The quantity $\sigma(\Omega)$, which depends only on the geometry of $\Omega$, will play an important role in the sequel. Throughout the paper we will suppose that $\Omega$ satisfies the following condition:

Assumption 2.1. The domain $\Omega \subset \mathbb{R}^{\mathrm{d}}$ is open bounded and such that

$$
\inf _{u \in H_{0}^{1}(\Omega)} \frac{\int_{\Omega}|\nabla u|^{2}}{\int_{\Omega}|u|^{2} / \delta^{2}}=: c_{h}^{-1}(\Omega)>0 .
$$

Note that $c_{h}(\Omega)$ is the best constant in the Hardy inequality

$$
\int_{\Omega} \frac{|u(x)|^{2}}{\delta(x)^{2}} d x \leq c_{h}(\Omega) \int_{\Omega}|\nabla u(x)|^{2} d x \quad \forall u \in H_{0}^{1}(\Omega) .
$$

Remark 2.2. Assumption 2.1 is satisfied, for example, for all open bounded domains with Lipschitz boundary, see [1]. It is know that for simply connected planar domains $c_{h}(\Omega) \leq 16$, [1], and for convex domains $\mathrm{c}_{\mathrm{h}}(\Omega)=4$, see e.g. [3, 19], 


\subsection{Main results: Dirichlet Laplacian.}

Theorem 2.3. For any $\mathrm{N} \in \mathbb{N}$ we have

$$
\sum_{j=1}^{N} \lambda_{j}(\Omega) \geq C_{d}|\Omega|^{-\frac{2}{d}} N^{1+\frac{2}{d}}+\frac{1}{16 c_{h}(\Omega)} \frac{\sigma^{2}(\Omega)}{|\Omega|^{2}} N
$$

For convex domains, in particular, we have

Corollary 2.4. Let $\Omega \subset \mathbb{R}^{\mathrm{d}}$ satisfy assumption 2.1 and suppose moreover that $\Omega$ is convex. Then for any $\mathrm{N} \in \mathbb{N}$ it holds

$$
\sum_{j=1}^{N} \lambda_{j}(\Omega) \geq C_{d}|\Omega|^{-\frac{2}{d}} N^{1+\frac{2}{d}}+\frac{N}{64 R_{i}^{2}(\Omega)}
$$

Remark 2.5. Let us compare the lower bound (2.6) with (1.5). Assume that $a \in \mathbb{R}^{\mathrm{d}}$ is such that $\mathrm{I}(\Omega)=\int_{\Omega}|x-a|^{2} \mathrm{~d} x$ and let $\mathrm{B}(\mathrm{a}, \mathrm{R})$ be the ball centered in a with radius $\mathrm{R}$ chosen such that $|B(a, R)|=|\Omega|$. Then it is easily seen that

$$
\mathrm{I}(\Omega) \geq \mathrm{I}(\mathrm{B}(\mathrm{a}, \mathrm{R}))=\frac{\mathrm{d}}{\mathrm{d}+2}|\Omega| \mathrm{R}^{2} .
$$

By using the fact that $R \geq R_{i}(\Omega)$ we thus obtain

$$
\frac{1}{R_{i}^{2}(\Omega)} \geq \frac{d}{d+2} \frac{|\Omega|}{I(\Omega)}
$$

Hence, for convex $\Omega$, inequality (2.6) implies (1.5) with $M_{d}=\frac{d}{64(d+2)}$. For $d \geq 3$ this is better than the lower bound $M_{d} \geq \frac{1}{24(d+2)}$ obtained in [20].

On the other hand, for domains which are wide in one direction and thin in another the estimate (2.6) is much sharper than (1.5) due to the fact that $\lambda_{1}(\Omega)$ is proportional to $R_{i}(\Omega)^{-2}$. Indeed, consider for example the rectangle $\Omega_{\varepsilon}=\left(0, \varepsilon^{-1}\right) \times(0, \varepsilon)$ in $\mathbb{R}^{2}$. Then as $\varepsilon \rightarrow 0$ we find $\left|\Omega_{\varepsilon}\right| / \mathrm{I}\left(\Omega_{\varepsilon}\right) \sim 3 \varepsilon^{2}$, while on the right hand side of (2.6) we have $\mathrm{R}_{i}^{-2}\left(\Omega_{\varepsilon}\right)=\varepsilon^{-2}$ which is of the same order of $\varepsilon$ as the left hand side.

Remark 2.6. The reminder terms in both bounds (2.6) and (1.5) are not sharp in the order of N. This follows from the refined Weyl asymptotic

$$
\sum_{j=1}^{N} \lambda_{j}(\Omega)=C_{d}|\Omega|^{-\frac{2}{d}} N^{1+\frac{2}{d}}+K_{d} \frac{|\partial \Omega|}{|\Omega|^{1+\frac{1}{d}}} N^{1+\frac{1}{d}}(1+o(1)) \quad N \rightarrow \infty
$$

with a positive constant $K_{d}$ depending only $d$. The asymptotic equation (2.8) was first proven by Ivrii [11, 12] for smooth domains under an additional assumption on the set of all periodic geodesic billiards in $\Omega$, see also [22]. Recently, (2.8) was extended to all domains with $C^{1, \alpha}$ boundary (with $\alpha>0)$ by Frank and Geisinger [6]. 
2.3. Main results: magnetic Dirichlet Laplacian. As already mentioned in the introduction, our approach enables us to extend the bound (2.5) to the magnetic Dirichlet Laplacian. Let $B \in \mathbb{R}$ be a non-zero constant define vector potential $A(x)=\frac{1}{2}\left(-B x_{2}, B x_{1}\right)$ so that curl $A=B$. We then have

Theorem 2.7. Let $\mathrm{d}=2$. Then For any $\mathrm{N} \in \mathbb{N}$ it holds

$$
\sum_{j=1}^{N} \lambda_{j}(\Omega ; A) \geq \frac{2 \pi N^{2}}{|\Omega|}+\frac{1}{16 c_{h}(\Omega)} \frac{\sigma^{2}(\Omega)}{|\Omega|^{2}} N .
$$

Corollary 2.8. Let $\Omega \subset \mathbb{R}^{2}$ be bounded and convex. Then

$$
\sum_{j=1}^{N} \lambda_{j}(\Omega ; A) \geq \frac{2 \pi N^{2}}{|\Omega|}+\frac{N}{64 R_{i}^{2}(\Omega)} .
$$

\section{Proofs of the main results}

3.1. Dirichlet Laplacian. Given $\Lambda>0$ we denote by

$$
n(\Lambda)=\operatorname{card}\left\{\lambda_{j}(\Omega): \lambda_{j}(\Omega)<\Lambda\right\}
$$

the counting function. Let $\left\{u_{j}\right\}_{j \in \mathbb{N}}$ be the set of eigenfunctions of $-\Delta_{\Omega}$ corresponding to the eigenvalues $\lambda_{j}(\Omega)$. We assume that the eigenfunctions are normalised in $L^{2}(\Omega)$ and denote by $\hat{u}_{j}(\xi)$ the Fourier transform of $u_{j}$ extended by zero to $\mathbb{R}^{d}$;

$$
\hat{u}_{j}(\xi)=(2 \pi)^{-d / 2} \int_{\Omega} e^{-i x \cdot \xi} u_{j}(x) d x
$$

Then

$$
\begin{aligned}
\sum_{j: \lambda_{j}(\Omega)<\Lambda}\left(\Lambda-\lambda_{j}(\Omega)\right)= & \sum_{j \leq n(\Lambda)} \int_{\Omega}\left(\Lambda\left|u_{j}(x)\right|^{2}-\left|\nabla u_{j}(x)\right|^{2}\right) d x=\sum_{j \leq n(\Lambda)} \int_{\mathbb{R}^{d}}\left(\Lambda-|\xi|^{2}\right)\left|\hat{u}_{j}(\xi)\right|^{2} d \xi \\
= & \sum_{j \in \mathbb{N}} \int_{\mathbb{R}^{d}}\left(\Lambda-|\xi|^{2}\right)_{+}\left|\hat{u}_{j}(\xi)\right|^{2} d \xi \\
& \quad-\int_{\mathbb{R}^{d}}\left(|\xi|^{2}-\Lambda\right)_{+} R_{1}(\Lambda, \xi) d \xi-\int_{\mathbb{R}^{d}}\left(\Lambda-|\xi|^{2}\right)_{+} R_{2}(\Lambda, \xi) d \xi
\end{aligned}
$$

where

$$
R_{1}(\Lambda, \xi)=\sum_{j \leq n(\Lambda)}\left|\hat{u}_{j}(\xi)\right|^{2}, \quad R_{2}(\Lambda, \xi)=\sum_{j>n(\Lambda)}\left|\hat{u}_{j}(\xi)\right|^{2} .
$$

Since $\left\{u_{j}\right\}_{j \in \mathbb{N}}$ is an orthonormal basis of $L^{2}(\Omega)$ and $\left\|e^{-i x \cdot \xi}\right\|_{L^{2}(\Omega)}^{2}=|\Omega|$, the Parseval identity implies

$$
\mathrm{R}_{1}(\Lambda, \xi)+\mathrm{R}_{2}(\Lambda, \xi)=\sum_{j \in \mathbb{N}}\left|\hat{\iota}_{j}(\xi)\right|^{2}=(2 \pi)^{-\mathrm{d}}|\Omega| \quad \forall \xi \in \mathbb{R}^{\mathrm{d}} .
$$

Note also that, by the Pythagoras theorem, we have

$$
\mathrm{R}_{2}(\Lambda, \xi)=(2 \pi)^{-\mathrm{d}} \int_{\Omega}\left|e^{-i x \cdot \xi}-(2 \pi)^{\mathrm{d} / 2} \sum_{j \leq \mathfrak{n}(\Lambda)} \hat{u}_{j}(\xi) u_{j}(x)\right|^{2} d x .
$$


Our aim is to estimate $R_{2}(\Lambda . \xi)$ from below by a function of $\Lambda$, uniformly in $\xi$. Since $|a-b|^{2} \geq$ $\frac{1}{2}|a|^{2}-|b|^{2}$ for all $a, b \in \mathbb{C}$, from (3.4) it follows that for any $\beta>0$

$$
\begin{aligned}
\mathrm{R}_{2}(\Lambda, \xi) & \geq(2 \pi)^{-\mathrm{d}} \int_{\Omega_{\beta}}\left|e^{-i x \cdot \xi}-(2 \pi)^{\mathrm{d} / 2} \sum_{j \leq n(\Lambda)} \hat{u}_{j}(\xi) u_{j}(x)\right|^{2} \mathrm{~d} x \\
& \geq \frac{1}{2}(2 \pi)^{-\mathrm{d}}\left|\Omega_{\beta}\right|-\int_{\Omega_{\beta}}\left|\mathrm{F}_{\Lambda}(\xi, x)\right|^{2} \mathrm{~d} x,
\end{aligned}
$$

where we used the shorthand

$$
F_{\Lambda}(\xi, x)=\sum_{j \leq n(\Lambda)} \widehat{u}_{j}(\xi) u_{j}(x) .
$$

Since $F_{\Lambda}(\xi, \cdot) \in H_{0}^{1}(\Omega)$ for each $\Lambda>0$ and each $\xi \in \mathbb{R}^{d}$, the Hardy inequality (2.4) in combination with (3.3) gives

$$
\begin{aligned}
\int_{\Omega_{\beta}}\left|\mathrm{F}_{\Lambda}(\xi, x)\right|^{2} \mathrm{~d} x & \leq \beta^{2} \int_{\Omega_{\beta}} \frac{\left|\mathrm{F}_{\Lambda}(\xi, x)\right|^{2}}{\delta^{2}(x)} \mathrm{d} x \leq \beta^{2} \int_{\Omega} \frac{\left|\mathrm{F}_{\Lambda}(\xi, x)\right|^{2}}{\delta^{2}(x)} \mathrm{d} x \\
& \leq \beta^{2} c_{h}(\Omega) \int_{\Omega}\left|\nabla_{x} \mathrm{~F}_{\Lambda}(\xi, x)\right|^{2} \mathrm{~d} x=\beta^{2} c_{h}(\Omega) \sum_{j \leq \mathfrak{n}(\Lambda)} \lambda_{j}(\Omega)\left|\hat{u}_{j}(\xi)\right|^{2} \\
& \leq \beta^{2} \wedge c_{h}(\Omega)(2 \pi)^{-\mathrm{d}}|\Omega| .
\end{aligned}
$$

Hence in view of (3.5) and (3.6) we get

$$
\mathrm{R}_{2}(\Lambda, \xi) \geq(2 \pi)^{-\mathrm{d}}\left(\frac{1}{2} \frac{\left|\Omega_{\beta}\right|}{\beta}-\Lambda \beta \mathrm{c}_{\mathrm{h}}(\Omega)|\Omega|\right) \beta .
$$

Now we choose

$$
\beta=\frac{\sigma(\Omega)}{4 c_{h}(\Omega)|\Omega|} \Lambda^{-1}
$$

where $c_{h}(\Omega)$ is the constant from the Hardy inequality (2.4). Note that the latter implies

$$
\lambda_{1}(\Omega) \geq \frac{1}{c_{h}(\Omega) R_{i}^{2}(\Omega)} .
$$

Using the definition of $\sigma(\Omega)$ we then find that for any $\Lambda \geq \lambda_{1}(\Omega)$ it holds

$$
\beta \leq \frac{\sigma(\Omega)}{4 c_{h}(\Omega)|\Omega|} \lambda_{1}^{-1}(\Omega) \leq \frac{1}{4 c_{h}(\Omega) R_{i}(\Omega)} \lambda_{1}^{-1}(\Omega) \leq \frac{R_{i}(\Omega)}{4} .
$$

From (2.2) it thus follows that with our choice of $\beta$ we have

$$
\frac{\left|\Omega_{\beta}\right|}{\beta} \geq \sigma(\Omega)
$$

Inserting the above estimate together with (3.8) into (3.7) we obtain

$$
\mathrm{R}_{2}(\Lambda, \xi) \geq \frac{1}{16 c_{h}(\Omega)}(2 \pi)^{-\mathrm{d}} \frac{\sigma^{2}(\Omega)}{|\Omega|} \Lambda^{-1} .
$$


Proposition 3.1. For any $\Lambda \geq \lambda_{1}(\Omega)$ it holds

$$
\sum_{j: \lambda_{j}(\Omega)<\Lambda}\left(\Lambda-\lambda_{j}(\Omega)\right) \leq L_{1, d}^{c l}|\Omega| \Lambda^{1+\frac{d}{2}}-\frac{L_{1, d}^{c l}}{16 c_{h}(\Omega)} \frac{\sigma^{2}(\Omega)}{|\Omega|} \Lambda^{\frac{d}{2}}
$$

where

$$
\mathrm{L}_{1, \mathrm{~d}}^{\mathrm{cl}}=\frac{1}{2^{\mathrm{d}} \pi^{\mathrm{d} / 2} \Gamma(2+\mathrm{d} / 2)}
$$

Proof. Since $R_{1}(\Lambda, \xi) \geq 0$, equations (3.2) and (3.3) imply

$$
\sum_{j: \lambda_{j}(\Omega)<\Lambda}\left(\Lambda-\lambda_{j}(\Omega)\right) \leq(2 \pi)^{-\mathrm{d}}|\Omega| \int_{\mathbb{R}^{\mathrm{d}}}\left(\Lambda-|\xi|^{2}\right)_{+} \mathrm{d} \xi-\int_{\mathbb{R}^{\mathrm{d}}}\left(\Lambda-|\xi|^{2}\right)_{+} \mathrm{R}_{2}(\Lambda, \xi) \mathrm{d} \xi .
$$

The claim now follows by inserting the lower bound (3.11) and integrating with respect to $\xi$.

Note that the right hand side of (3.12) is positive for all $\Lambda \geq \lambda_{1}(\Omega)$ in view of inequality (3.9) and $\sigma(\Omega) \leq|\Omega| / R_{i}(\Omega)$.

Proof of Theorem 2.3. From (3.12) it follows that

$$
\begin{aligned}
\sum_{j: \lambda_{j}(\Omega)<\Lambda}\left(\Lambda-\lambda_{j}(\Omega)\right) & \leq L_{1, d}^{c l}|\Omega| \Lambda^{1+\frac{d}{2}}\left(1-\frac{1}{16 c_{h}(\Omega)} \frac{\sigma^{2}(\Omega)}{|\Omega|^{2} \Lambda}\right) \\
& \leq L_{1, d}^{c l}|\Omega| \Lambda^{1+\frac{d}{2}}\left(1-\frac{1}{16 c_{h}(\Omega)} \frac{\sigma^{2}(\Omega)}{|\Omega|^{2} \Lambda}\right)^{1+\frac{d}{2}} \\
& =L_{1, d}^{c l}|\Omega|\left(\Lambda-\frac{1}{16 c_{h}(\Omega)} \frac{\sigma^{2}(\Omega)}{|\Omega|^{2}}\right)^{1+\frac{d}{2}} .
\end{aligned}
$$

Since both sides of the above inequality are convex functions of $\Lambda$, we can apply the Legendre transform. This yields (2.5).

\section{Convex domains.}

Lemma 3.2. Let $\Omega \subset \mathbb{R}^{\mathrm{d}}$ be bounded and convex. Then

$$
\sigma(\Omega)=\frac{|\Omega|}{R_{i}(\Omega)} .
$$

Proof. Let us first prove the statement for domains with $\mathrm{C}^{1}$ boundary. We are going to show that

$$
f(\beta)=\frac{\left|\Omega_{\beta}\right|}{\beta}
$$

is a decreasing function of $\beta$ on $\left(0, R_{i}(\Omega)\right)$. To this end let $\beta_{0} \in\left(0, R_{i}(\Omega)\right)$ and consider the sets

$$
E_{0}=\left\{x \in \Omega: \delta(x) \geq \beta_{0}\right\}, \quad \text { and } \quad E_{t}=\left\{x \in \Omega \backslash E_{0}: \operatorname{dist}\left(x, E_{0}\right) \leq t\right\}, \quad t>0 .
$$

From the convexity of $\Omega$ it follows that $\delta$ is concave and therefore $E_{0}$ is a compact convex set. Hence by the Steiner formula, see e.g. [10], it holds

$$
\left|E_{t}\right|=\sum_{j=0}^{d} K_{j}\left(E_{0}\right) t^{j}
$$


where $K_{j}\left(E_{0}\right)$ are non-negative coefficients depending on the geometry of $E_{0}$. We claim that

$$
\mathrm{E}_{\beta_{0}-\beta} \cup \mathrm{E}_{0}=\Omega_{\beta}^{c}, \quad 0<\beta<\beta_{0},
$$

where $\Omega_{\beta}^{c}=\Omega \backslash \Omega_{\beta}$ is the complement of $\Omega_{\beta}$ in $\Omega$. Indeed, let $y \in \partial E_{0}$ and denote by $r_{y}$ the half-line emanating from $y$ perpendicularly to the tangent plane of $\partial \mathrm{E}_{0}$ at $\mathrm{y}$. Let $z_{\mathrm{y}} \in \partial \Omega$ be given by the intersection of $\partial \Omega$ and $r_{y}$. Since $\delta(y)=\beta_{0}$ we have

$$
\operatorname{dist}\left(y, z_{y}\right)=\delta(y)=\beta_{0}, \quad y \in \partial E_{0} .
$$

Now let $x \in \Omega_{\beta}^{c}$. Then there exists an $y(x) \in \partial E_{0}$ such that $x \in r_{y(x)}$. Hence

$$
\operatorname{dist}(y(x), x)=\delta(y(x))-\operatorname{dist}\left(x, z_{y(x)}\right)=\beta_{0}-\operatorname{dist}\left(x, z_{y(x)}\right) \leq \beta_{0}-\delta(x) \leq \beta_{0}-\beta .
$$

This implies that $\Omega_{\beta}^{c} \subseteq \mathrm{E}_{\beta_{0}-\beta} \cup \mathrm{E}_{0}$. To prove the opposite inclusion, let $x \in\left(\mathrm{E}_{\beta_{0}-\beta} \cup \mathrm{E}_{0}\right)$. By the triangle inequality and (3.17)

$$
\beta_{0} \leq \operatorname{dist}\left(x, E_{0}\right)+\delta(x) \leq \beta_{0}-\beta+\delta(x)
$$

which shows that $x \in \Omega_{\beta}^{c}$. Therefore (3.16) holds true and consequently

$$
\left|\Omega_{\beta}\right|=|\Omega|-\left|E_{\beta_{0}-\beta} \cup E_{0}\right| \text {. }
$$

In view of (3.15) it follows that $\left|E_{\beta_{0}-\beta} \cup E_{0}\right|$ is a convex function of $\beta$. Hence $\left|\Omega_{\beta}\right|$ is a concave function of $\beta$ on $\left(0, \beta_{0}\right)$, see $(3.18)$, and since $\left|\Omega_{0}\right|=0$, we easily verify that $f(\beta)=\left|\Omega_{\beta}\right| / \beta$ is decreasing on $\left(0, \beta_{0}\right)$ for any $\beta_{0}<R_{i}(\Omega)$. This proves the statement of the Lemma for $C^{1}$ smooth domains.

If $\partial \Omega$ is not $C^{1}$, then we approximate $\Omega$ by a sequence of domains $\Omega^{n}$ with $C^{1}$ smooth boundary and such that the Hausdorf distance between $\Omega$ and $\Omega^{n}$ tends to zero as $n \rightarrow \infty$. Then

$$
f(\beta)=\lim _{n \rightarrow \infty} \frac{\left|\Omega_{\beta}^{n}\right|}{\beta} .
$$

Since a pointwise limit of a sequence of decreasing functions is a decreasing function, we again conclude that $f(\beta)$ is decreasing. This completes the proof.

Proof of Corollary 2.4 The claim follows from Theorem 2.3, Lemma 3.2 and the fact that for convex domains $c_{h}(\Omega)=4$ independently of $\Omega,[3,19]$.

3.2. Magnetic Dirichlet Laplacian. Let $P_{k}$ be the orthogonal projection onto the kth Landau level $\mathrm{B}(2 \mathrm{k}-1)$ of the Landau Hamiltonian with constant magnetic field $\mathrm{B}$ in $\mathrm{L}^{2}\left(\mathbb{R}^{2}\right)$. Denote by $\mathrm{P}_{\mathrm{k}}(x, y)$ the integral kernel of $P_{k}$. Note that

$$
\begin{aligned}
P_{k}(x, x) & =\frac{1}{2 \pi} B \\
\int_{\mathbb{R}^{2}}\left(\int_{\Omega}\left|P_{k}(y, x)\right|^{2} d x\right) d y & =\int_{\Omega}\left(\int_{\mathbb{R}^{2}} P_{k}(y, x) \overline{P_{k}(x, y)} d y\right) d x \\
& =\int_{\Omega} P_{k}(x, x) d x=\frac{B}{2 \pi}|\Omega| .
\end{aligned}
$$

Let $\phi_{j}$ be the normalised eigenfunctions of $H_{\Omega}(A)$ corresponding to the eigenvalues $\lambda_{j}(\Omega ; A)$. Put

$$
f_{k, j}(y)=\int_{\Omega} P_{k}(y, x) \phi_{j}(x) d x, \quad y \in \mathbb{R}^{2} .
$$


Our goal is to establish an analog of Proposition 3.1 for magnetic Dirichlet Laplacians on planar domains. Let $\Lambda>0$. We have

$$
\begin{aligned}
\sum_{j: \lambda_{j}(\Omega ; A)<\Lambda}\left(\Lambda-\lambda_{j}(\Omega ; A)\right) & =\sum_{j: \lambda_{j}(\Omega ; A)<\Lambda}\left(\Lambda\left\|\phi_{j}\right\|_{L^{2}(\Omega)}^{2}-\left\|\left(i \nabla_{x}+A\right) \phi_{j}\right\|_{L^{2}(\Omega)}^{2}\right) \\
& =\sum_{j: \lambda_{j}(\Omega ; A)<\Lambda} \sum_{k \in \mathbb{N}}\left(\Lambda\left\|f_{k, j}\right\|_{L^{2}\left(\mathbb{R}^{2}\right)}^{2}-\left\|\left(i \nabla_{x}+A\right) f_{k, j}\right\|_{L^{2}\left(\mathbb{R}^{2}\right)}^{2}\right) \\
& =\sum_{j: \lambda_{j}(\Omega ; A} \sum_{k}(\Lambda-B(2 k-1))\left\|f_{k, j}\right\|_{L^{2}\left(\mathbb{R}^{2}\right)}^{2} .
\end{aligned}
$$

In analogy with the procedure in the non-magnetic case we split

$$
\begin{aligned}
\sum_{j: \lambda_{j}(\Omega ; A)<\Lambda}\left(\Lambda-\lambda_{j}(\Omega ; A)\right) & =\sum_{j: \lambda_{j}(\Omega ; A)<\Lambda} \sum_{k \in \mathbb{N}}(\Lambda-B(2 k-1))\left\|f_{k, j}\right\|_{L^{2}\left(\mathbb{R}^{2}\right)}^{2} \\
& =\sum_{j: \lambda_{j}(\Omega ; A)<\Lambda} \sum_{k: \Lambda>B(2 k-1)}(\Lambda-B(2 k-1))\left\|f_{k, j}\right\|_{L^{2}\left(\mathbb{R}^{2}\right)}^{2} \\
& +\sum_{j: \lambda_{j}(\Omega ; A)<\Lambda} \sum_{k: \Lambda \leq B(2 k-1)}(\Lambda-B(2 k-1))\left\|f_{k, j}\right\|_{L^{2}\left(\mathbb{R}^{2}\right)}^{2} \\
& =\sum_{k: \Lambda>B(2 k-1)}(\Lambda-B(2 k-1)) \sum_{j \in \mathbb{N}}\left\|f_{k, j}\right\|_{L^{2}\left(\mathbb{R}^{2}\right)}^{2} \\
& -\sum_{k: \Lambda \leq B(2 k-1)}(B(2 k-1)-\Lambda) R_{1}(\Lambda, k) \\
& -\sum_{k: \Lambda>B(2 k-1)}(\Lambda-B(2 k-1)) R_{2}(\Lambda, k),
\end{aligned}
$$

where

$$
R_{1}(\Lambda, k)=\sum_{j: \lambda_{j}(\Omega ; A)<\Lambda}\left\|f_{k, j}\right\|_{L^{2}\left(\mathbb{R}^{2}\right)}^{2}, \quad R_{2}(\lambda, k)=\sum_{j: \lambda_{j}(\Omega ; A) \geq \Lambda}\left\|f_{k, j}\right\|_{L^{2}\left(\mathbb{R}^{2}\right)}^{2} .
$$

By Parseval's identity and equation (3.20) it follows that for all $\Lambda>0$ and all $k \in \mathbb{N}$ we have

$$
\begin{aligned}
\sum_{j \in \mathbb{N}}\left\|f_{k, j}\right\|_{L^{2}\left(\mathbb{R}^{2}\right)}^{2} & =R_{1}(\Lambda, k)+R_{2}(\Lambda, k)=\int_{\mathbb{R}^{2}}\left|\sum_{j \in \mathbb{N}} \int_{\Omega} P_{k}(y, x) \phi_{j}(x) d x\right|^{2} d y \\
& =\int_{\mathbb{R}^{2}} \int_{\Omega}\left|P_{k}(y, x)\right|^{2} d x d y=\frac{B}{2 \pi}|\Omega|
\end{aligned}
$$

Let

$$
Q_{k}(x, y ; \Lambda)=\sum_{j: \lambda_{j}(\Omega ; A)<\Lambda} f_{k, j}(y) \bar{\phi}_{j}(x) .
$$


We now use identities 3.19 - (3.20) to find that, similarly as in section 3 for any $\beta \leq R_{i}(\Omega)$ it holds

$$
\begin{aligned}
R_{2}(\Lambda, k) & =\int_{\mathbb{R}^{2}}\left(\int_{\Omega}\left|P_{k}(x, y)-Q_{k}(x, y ; \Lambda)\right|^{2} d x\right) d y \\
& \geq \frac{1}{2} \int_{\mathbb{R}^{2}} \int_{\Omega_{\beta}}\left|P_{k}(x, y)\right|^{2} d x d y-\int_{\mathbb{R}^{2}} \int_{\Omega_{\beta}}\left|Q_{k}(x, y ; \Lambda)\right|^{2} d x d y \\
& =\frac{B}{4 \pi}\left|\Omega_{\beta}\right|-\int_{\mathbb{R}^{2}} \int_{\Omega_{\beta}}\left|Q_{k}(x, y ; \Lambda)\right|^{2} d x d y .
\end{aligned}
$$

Since $\mathrm{Q}_{k}(\cdot, y ; \Lambda) \in \mathrm{H}_{0}^{1}(\Omega)$ for all $k \in \mathbb{N}, y \in \mathbb{R}^{2}$ and $\Lambda>0$, the Hardy inequality (2.4) in combination with (1.7) yield

$$
\begin{aligned}
\int_{\Omega_{\beta}}\left|Q_{k}(x, y ; \Lambda)\right|^{2} d x & \leq \beta^{2} \int_{\Omega_{\beta}} \frac{\left|Q_{k}(x, y ; \Lambda)\right|^{2}}{\delta^{2}(x)} \mathrm{d} x \leq \beta^{2} \int_{\Omega} \frac{\left|Q_{k}(x, y ; \Lambda)\right|^{2}}{\delta^{2}(x)} d x \\
& \leq \beta^{2} c_{h}(\Omega) \int_{\Omega}\left|\left(i \nabla_{x}+A\right) Q_{k}(x, y ; \Lambda)\right|^{2} d x \\
& =\beta^{2} c_{h}(\Omega) \sum_{j: \lambda_{j}(\Omega ; A)<\Lambda}\left|f_{k, j}(y)\right|^{2} \lambda_{j}(\Omega ; A) \leq \beta^{2} c_{h}(\Omega) \Lambda \sum_{j: \lambda_{j}(\Omega ; A)<\Lambda}\left|f_{k, j}(y)\right|^{2} .
\end{aligned}
$$

By inserting the above estimate into (3.24) and using (3.22) again we obtain

$$
\begin{aligned}
R_{2}(\Lambda, k) & \geq \frac{B}{4 \pi}\left|\Omega_{\beta}\right|-\beta^{2} c_{h}(\Omega) \wedge \sum_{j: \lambda_{j}(\Omega ; A)<\Lambda}\left\|f_{k, j}\right\|_{L^{2}\left(\mathbb{R}^{2}\right)}^{2} \\
& \geq \frac{B}{4 \pi}\left(\frac{\left|\Omega_{\beta}\right|}{\beta}-2 \beta \Lambda c_{h}(\Omega)|\Omega|\right) \beta .
\end{aligned}
$$

Note that in view of (1.7) we have

$$
\lambda_{1}(\Omega ; A) \geq \lambda_{1}(\Omega) .
$$

Hence choosing $\beta$ as in (3.8) and following the reasoning in (3.10) we conclude that $\beta \leq R_{\mathfrak{i}}(\Omega) / 4$ and therefore $\frac{\left|\Omega_{\beta}\right|}{\beta} \geq \sigma(\Omega)$. This implies

$$
\mathrm{R}_{2}(\Lambda, k) \geq \frac{\mathrm{B}}{32 \pi \mathrm{c}_{\mathrm{h}}(\Omega)} \frac{\sigma^{2}(\Omega)}{|\Omega|} \Lambda^{-1} \quad \forall k \in \mathbb{N} .
$$

Proposition 3.3. Let $\mathrm{d}=2$. For any $\Lambda \geq \lambda_{1}(\Omega ; \mathrm{A})$ it holds

$$
\sum_{j: \lambda_{j}(\Omega ; A)<\Lambda}\left(\Lambda-\lambda_{j}(\Omega ; A)\right) \leq \frac{|\Omega|}{8 \pi} \Lambda^{2}-\frac{1}{128 \pi c_{h}(\Omega)} \frac{\sigma^{2}(\Omega)}{|\Omega|} \Lambda .
$$

Proof. Put $M=\left[\frac{\Lambda}{2 B}+\frac{1}{2}\right]$ and $m=\left\{\frac{\Lambda}{2 B}+\frac{1}{2}\right\}$ and thus $M+m=\frac{\Lambda}{2 B}+\frac{1}{2}$. Then

$$
\begin{aligned}
\sum_{k: \Lambda>B(2 k-1)}(\Lambda-B(2 k-1)) & =M \wedge-B M^{2}=B M\left(\frac{\Lambda}{B}-M\right) \\
& =B\left(\frac{\Lambda}{2 B}+\frac{1}{2}-m\right)\left(\frac{\Lambda}{2 B}-\frac{1}{2}+m\right) \\
& =B\left(\frac{\Lambda^{2}}{4 B^{2}}-\left(\frac{1}{2}-m\right)^{2}\right) .
\end{aligned}
$$


Since $R_{1}(\Lambda, k) \geq 0$, the above identity together with (3.21) and (3.22) implies

$$
\begin{aligned}
\sum_{j: \lambda_{j}(\Omega ; A)<\Lambda}\left(\Lambda-\lambda_{j}(\Omega ; A)\right) \leq & \frac{|\Omega|}{8 \pi} \Lambda^{2}-\frac{1}{128 c_{h}(\Omega) \pi} \frac{\sigma^{2}(\Omega)}{|\Omega|} \Lambda \\
& -B^{2}\left(\frac{1}{2}-m\right)^{2}\left(\frac{|\Omega|}{2 \pi}-\frac{1}{32 \pi c_{h}(\Omega)} \frac{\sigma^{2}(\Omega)}{|\Omega| \Lambda}\right) .
\end{aligned}
$$

The last term on the right hand side of the last inequality is negative since $\Lambda c_{h}(\Omega) \geq$ $\lambda_{1}(\Omega) c_{h}(\Omega) \geq R_{i}^{-2}(\Omega)$, by (2.4) and (3.25), and $\sigma(\Omega) \leq|\Omega| / R_{i}(\Omega)$. The claim now follows.

Proof of Theorem 2.7 Inequality (2.9) now follows from Proposition 3.3 by the Legendre transformation in the same as in the case of the Dirichlet Laplacian.

Corollary 2.8 is a consequence of Theorem 2.7 and Lemma 3.2 .

\section{Further improvements}

The order of the reminder term in (3.27) can further be improved applying a straightforward generalization of a result by Davies, [4]. We are grateful to Rupert Frank who pointed this fact out to us.

Proposition 4.1. Let $\Omega \subset \mathbb{R}^{\mathrm{d}}$ be an open bounded set. Let $\mathrm{A} \in \mathrm{C}\left(\bar{\Omega}, \mathbb{R}^{2}\right)$ and let $\mathrm{H}(\mathrm{A})$ be the associated magnetic Dirichlet Lalpacian in $\mathrm{L}^{2}(\Omega)$. Assume that the Hardy inequality

$$
\int_{\Omega}|i \nabla u+A u|^{2} d x \geq c^{-2} \int_{\Omega} \frac{|u|^{2}}{\delta^{2}} d x, \quad u \in C_{0}^{\infty}(\Omega)
$$

holds for some $\mathrm{c} \geq 2$. Then for every $\beta>0$,

$$
\int_{\Omega_{\beta}}|u|^{2} d x \leq c^{2+2 / c} \beta^{2+2 / c}\|H(A) u\|\left\|H(A)^{1 / c} u\right\|
$$

for any $\mathrm{u}$ in the operator domain of $\mathrm{H}(\mathrm{A})$.

Proposition 4.1 was proved in [4] for the case $A=0$. However, a detailed inspection of the proof of [4, Thm. 4] shows that the same method applies also to the magnetic Dirichlet Laplacian. We then have

Theorem 4.2. Let $\Omega \subset \mathbb{R}^{2}$ be open and bounded. Let $\mathrm{A}=\frac{1}{2}\left(-\mathrm{B} x_{2}, \mathrm{~B} x_{1}\right)$. Then for any $\Lambda \geq$ $\lambda_{1}(\Omega ; A)$ it holds

$$
\sum_{j: \lambda_{j}(\Omega ; A)<\Lambda}\left(\Lambda-\lambda_{j}(\Omega ; A)\right) \leq \frac{|\Omega|}{8 \pi} \Lambda^{2}-K(\Omega) \sigma(\Omega)\left(\frac{\sigma(\Omega)}{|\Omega|}\right)^{\frac{2}{2+\mu}} \Lambda^{\frac{3+\mu}{2+\mu}}
$$

where $\mu=\mu(\Omega)=\sqrt{c_{h}(\Omega)}$ and

$$
\mathrm{K}(\Omega)=\frac{2+\mu}{16 \pi \mu}(2+2 \mu)^{-\frac{2+3 \mu}{2+\mu}}
$$


Proof. Let us fix $k \in \mathbb{N}$ and $y \in \mathbb{R}^{2}$. Since $Q_{k}(\cdot, y ; \Lambda)$ belongs to the domain of $H(A)$ for any $\Lambda>0$, see equation (3.23), we can apply inequality (4.2), with $c=\mu=\sqrt{c_{h}(\Omega)}$, to the function $u=Q_{k}(\cdot, y ; \Lambda)$. This yields

$$
\int_{\Omega_{\beta}}\left|Q_{k}(x, y ; \Lambda)\right|^{2} d x \leq(\mu \beta)^{2+\frac{2}{\mu}} \Lambda^{1+\frac{1}{\mu}} \sum_{j: \lambda_{j}(\Omega ; A)<\Lambda}\left|f_{k, j}(y)\right|^{2} .
$$

If we now insert the above bound into (3.24) and keep in mind that

$$
\sum_{j: \lambda_{j}(\Omega ; A)<\Lambda}\left\|f_{k, j}\right\|_{L^{2}\left(\mathbb{R}^{2}\right)}^{2} \leq \frac{B}{2 \pi}|\Omega|
$$

by (3.22), we find that

$$
\mathrm{R}_{2}(\Lambda, k) \geq \frac{\mathrm{B}}{4 \pi}\left(\frac{\left|\Omega_{\beta}\right|}{\beta}-2 \mu^{2+\frac{2}{\mu}} \beta^{1+\frac{2}{\mu}} \Lambda^{1+\frac{1}{\mu}}|\Omega|\right) \beta .
$$

Optimizing the right hand side with respect to $\beta$ gives

$$
\mathrm{R}_{2}(\Lambda, k) \geq B K(\Omega) \sigma(\Omega)\left(\frac{\sigma(\Omega)}{|\Omega|}\right)^{\frac{\mu}{2+\mu}} \Lambda^{-\frac{1+\mu}{2+\mu}} .
$$

We now follow the arguments of the proof of Proposition 3.3 with the lower bound (3.26) replaced by (4.4) and arrive at (4.3).

Remark 4.3. The power of $\Lambda$ in the reminder term of (4.3) is larger than the one of (3.27) by factor $\frac{1}{2+\mu}$.

For convex domains inequality (4.1) holds true with $\mathrm{c}=2$. Hence Theorem 4.2 in combination with Lemma 3.2 implies

Corollary 4.4. Let $\Omega \subset \mathbb{R}^{2}$ be bounded and convex and let $\mathrm{A}=\frac{1}{2}\left(-\mathrm{B} x_{2}, \mathrm{~B} x_{1}\right)$. Then

$$
\sum_{j: \lambda_{j}(\Omega ; A)<\Lambda}\left(\Lambda-\lambda_{j}(\Omega ; A)\right) \leq \frac{|\Omega|}{8 \pi}\left(\Lambda^{2}-\frac{\Lambda^{5 / 4}}{36 R_{i}(\Omega)^{3 / 2}}\right) .
$$

\section{Acknowledgements}

The work on this paper was initiated by the programme Hamiltonians in Magnetic Fields in fall 2012 at the Institut Mittag- Leffler, Djursholm, Sweden. H.K. has been partially supported by the MIURPRIN 2010-11 grant for the project "Calcolo delle Variazioni". T.W. has been partially supported by the DFG project WE 1964/4-1.

\section{REFERENCES}

[1] A. Ancona : On strong barriers and inequality of Hardy for domains in $\mathbb{R}^{n}$. J. London Math. Soc. 34 (1986), $274-290$.

[2] F.A. Berezin: Covariant and contravariant symbols of operators. Izv. Akad. Nauk SSSR Ser. Mat. 36 (1972) 11341167.

[3] E.B. Davies: A review of Hardy inequalities. The Maz'ya anniversary collection, Vol. 2, Oper. Theory Adv. Appl. Birkhäuser, Basel, 110 (1999) 55-67.

[4] E. B. Davies: Sharp boundary estimates for elliptic operators. Math. Proc. Camb. Phil. Soc.129 (2000) 165-178.

[5] L. Erdös, M. Loss and V. Vougalter: Diamagnetic behaviour of sums of Dirichlet eigenvalues. Ann. Inst. Fourier 50 (2000) 891-907. 
[6] R. Frank, L. Geisinger: Two-term spectral asymptotics for the Dirichlet Laplacian on a bounded domain. In: Mathematical results in quantum physics, Proceedings of the QMath11 Conference, P. Exner (ed.), World Scientific, Singapore, 2011, 138-147.

[7] R. Frank, M. Loss and T. Weidl: Polya's conjecture in the presence of a constant magnetic field. J. Eur. Math. Soc. (JEMS) 11 (2009) 1365-1383.

[8] J.K. Freericks, E.H. Lieb, D. Ueltschi: Segregation in the Falicov-Kimball model. Comm. Math. Phys. 227 (2002) 243-279.

[9] L. Geisinger, A. Laptev and T. Weidl: Geometrical versions of improved Berezin-Li-Yau inequalities. J. Spectr. Theory 1 (2011) 87-109.

[10] M. Heveling, D. Hug, G. Last: Does polynomial parallel volume imply convexity? Math. Ann. 328 (2004) $469-479$.

[11] V. Ivrii: Microlocal analysis and precise spectral asymptotics.. Springer Monographs in Mathematics. SpringerVerlag, Berlin, 1998.

[12] V. Ivrii: The second term of the spectral asymptotics for a Laplace-Beltrami operator on manifolds with boundary. (Russian) Funktsional. Anal. i Prilozhen 14 (1980), no. 2, 25-34.

[13] H. Kovařík, S. Vugalter, T. Weidl : Two dimensional Berezin-Li-Yau inequalities with a correction term. Commun. Math. Phys. 287 (2009) 959-981.

[14] A. Laptev : Dirichlet and Neumann Eigenvalue Problems on Domains in Euclidean Spaces. J. Func. Anal. 151 (1997) 531-545.

[15] A. Laptev, T. Weidl : Sharp Lieb-Thirring inequalities in high dimensions. Acta Math. 184 (2000) 87-111.

[16] A. Laptev and T. Weidl: Hardy inequalities for magnetic Dirichlet forms. Oper. Theory Adv. Appl. 108 (1999) 299305.

[17] P. Li and S.T. Yau: On the Schrödinger equation and the eigenvalue problem. Comm. Math. Phys. 88, (1983) 309318.

[18] E. H. Lieb, M. Loss, Analysis. Second edition. Graduate Studies in Mathematics 14, American Mathematical Society, Providence, RI, 2001.

[19] T. Matskewich and P. E. Sobolevskii: The best possible constant in a generalized Hardy's inequality for convex domains in $\mathbb{R}^{n}$, Nonlinear Analysis TMA, 28 (1997), 1601-1610.

[20] A.D. Melas: A lower bound for sums of eigenvalues of the Laplacian. Proc. Amer. Math. Soc. 131 (2003) 631-636.

[21] G. Pólya: On the eigenvalues of vibrating membranes. Proc. London Math. Soc. 11 (1961) 419-433.

[22] Yu. Safarov, D. Vassiliev: The asymptotic distribution of eigenvalues of partial differential operators. Translations of Mathematical Monographs, 155. American Mathematical Society, Providence, RI, 1997.

[23] T. Weidl: Improved Berezin-Li-Yau inequalities with a remainder term. Amer. Math. Soc. Transl. 225 (2008) $253-$ 263.

[24] H. Weyl: Das asymptotische Verteilungsgesetz der Eigenwerte linearer partieller Differentialgleichungen. Math. Ann. 71 (1912) 441-479.

[25] S. Y. Yolcu: An improvement to a Berezin-Li-Yau type inequatliy. Proc. Amer. Math. Soc. 138 (2010), $4059-4066$.

[26] S. Y. Yolcu and T. Yolcu: A Berezin-Li-Yau type inequality for the fractional Laplacian on a bounded domain. Rev. Math. Phys. 24 (2012) 18 pp.

Hynek Kovařík, Dipartimento di Matematica, Università degli STUdi di Brescia, Via BranZe, 38 25123 BRESCIA, ITALY

E-mail address: hynek . kovarik@ing.unibs. it

Timo Weidl, Institute of Analysis, Dynamics And Modeling, Universität Stuttgart, PF 801140 , D-70569 STUTTGART, GERMANY

E-mail address: weidl@mathematik . uni-stuttgart.de 